\title{
IL PROGETTO DI RIFORMA SCOLASTICA DELL'ISTITUTO LOMBARDO (1848, RELATORE CARLO CATTANEO) E LA LEGGE CASATI (1859)
}

\author{
Nota del s.c. ANDREA SILVESTRI (*)
}

(Adunanza del 3 novembre 2011)

SuNTO. - In questo lavoro si presentano le linee fondamentali di due diversi documenti: un progetto di riforma di tutto l'insegnamento pubblico in Lombardia, elaborato dall'Istituto Lombardo e steso da Carlo Cattaneo alla vigilia delle Cinque Giornate; e la Legge che porta il nome di Gabrio Casati, la quale riordina l'intero sistema dell'istruzione dopo l'annessione della Lombardia al Piemonte (1859). Nel progetto del Lombardo si rilevano sia idee della più avanzata cultura milanese sia costanti del pensiero di Cattaneo: il quale è critico quando vedrà la luce la Legge Casati, anche per la sua ostilità alla soluzione filosabauda che aveva portato a questa Legge e all'Unità d'Italia. Ma nella struttura che nel 1863 Francesco Brioschi darà al Politecnico di Milano non mancano i segni della lezione cattaneana sull'istruzione scientifico-tecnico-produttiva.

$$
* * *
$$

ABSTRACT. - This essay describes the reasons of two different documents. The first one is the project of public teaching system in Lombardy developed by Istituto Lombardo and written by Carlo Cattaneo just before the uprising against Austrians of March 1848 socalled "Cinque Giornate di Milano". The second one is Casati Act, that reorganizes the whole educational system after the annexation of Lombardy into Piedmont in 1859. The project of 1848 was both inspired by the most advanced intellectuality of Milan and by the thought of Carlo Cattaneo, who was negative with Casati Act and with the whole unification process lead by Savoia court. But the structure that Francesco Brioschi gave in 1863 to the Politecnico di Milano (that had been established by Casati Act) was also influenced by the lesson of Cattaneo on scientific, technical and manufacturing education.

(*) Politecnico di Milano, Dipartimento di Energia, via La Masa 34, 20156 Milano, Italy. E-mail: andrea.silvestri@polimi.it 


\section{PREMESSA}

Nell'anno del centocinquantenario dell'Unità d'Italia, e anche nel 2013 del centocinquantenario del Politecnico di Milano, si è parlato di istruzione tecnico-scientifica, non senza riferimenti al progetto di riforma dell'Istituto Lombardo per l'insegnamento pubblico in Lombardia: il documento, steso come relatore da Carlo Cattaneo1843, fu travolto dalle Cinque Giornate, ma in parte fu ripreso (e questa volta con successo) da Cattaneo stesso per analoga iniziativa in Canton Ticino. La riflessione sulla formazione scolastica (come palestra di formazione civile, etica, politica) sarebbe stata una costante nel pensiero di Cattaneo, anche con quel tono perentorio e critico che gli è proprio.

Dopo l'annessione della Lombardia al Piemonte nel 1859, uno dei primi atti legislativi del regno sabaudo (ancora in regime di "pieni poteri”, con grande lungimiranza) fu la cosiddetta Legge Casati sulla pubblica istruzione - intervento impegnativo e suscettibile di critiche, in particolare da parte dello stesso Cattaneo come vedremo, ma intervento organico su tutto il sistema formativo, quale sarà poi solo la riforma Gentile del 1923.

Per il rilievo concettuale e premonitore del progetto di Cattaneo e per la portata innovativa, anche se non esente da limiti, della Legge Casati, si vuole qui fornire qualche elemento di confronto, mettendo in rilievo l'attenzione anche successiva (e anche polemica rispetto alla Legge Casati) di Cattaneo sull'istruzione in particolare tecnica.

\section{Il PROGETTO DELl'Istituto Lombardo E di CARLO CATTANEO}

Nel gennaio del 1848, anche per fronteggiare le spinte al rinnovamento che venivano dal Lombardo-Veneto, il governo austriaco aveva richiesto all'Istituto Lombardo di scienze, lettere ed arti ${ }^{1}$ un progetto di riforma dell'insegnamento elementare e ginnasiale in Lombardia.

1 La tradizione scientifica dell'Istituto Lombardo era ben radicata (primo presidente Alessandro Volta), e l'attenzione anche tecnico-produttiva era affidata sia a concorsi e premi per utili invenzioni sia soprattutto all'esistenza di un ricco gabinetto tecnologico, a disposizione di membri e soci ma anche aperto a chi volesse documentarsi e sperimentare sulla collezione di modelli, strumenti, apparecchi e macchine. 
Cattaneo (Fig. 1) - già autore della prima memorabile serie della rivista "Il Politecnico", 1839-1844 (Fig. 2), e poi impegnato nella fortunata esperienza di Relatore della Società d'Incoraggiamento d'Arti e Mestieri $^{2}$ (Fig. 3) - aveva coordinato da par suo una qualificatissima commissione di membri e soci, il cui lavoro risultò ben più generale del richiesto, riguardando tutto il sistema dell'istruzione, inclusa quella superiore. Il progetto fu presentato all'Istituto il 9 marzo ma Cattaneo operò ancora (ce ne sono prove documentarie) per recepire i commenti dei colleghi in vista della redazione finale. Finché il 18 marzo le Cinque Giornate irruppero freneticamente: il governo provvisorio confermò l'Istituto Lombardo, che risottopose il progetto alla Commissione. Ma il 6 agosto la rivoluzione fu domata con il ritorno degli austriaci, e poco dopo Cattaneo - già prima perplesso sulla tempestività della rivolta, e poi scontento della piega filosabauda che l'aristocrazia milanese aveva dato al governo provvisorio - si esiliò definitivamente in Ticino a Castagnola.

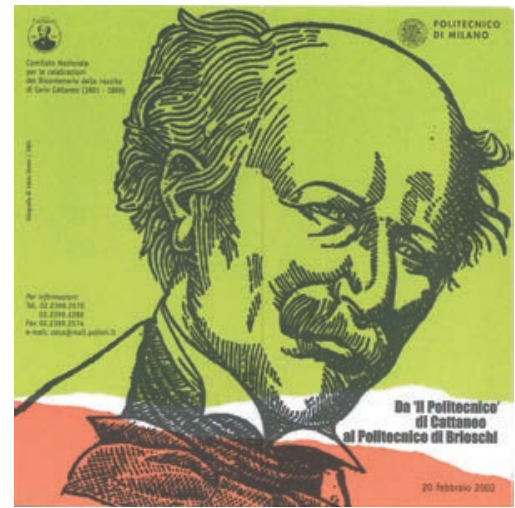

Fig. 1 - Carlo Cattaneo (1801-1869).

Alla nascita del Politecnico il gabinetto tecnologico sarebbe passato al Politecnico, come alcune collezioni pertinenti di Pavia. Sulla storia dell'Istituto Lombardo, cfr. E. Gatti, G. Orlandi, A. Robbiati Bianchi e M. Vitale (a cura di), L'Istituto Lombardo Accademia di Scienze e Lettere (Secoli XIX - XX), 3 voll., Milano, Istituto Lombardo Accademia di Scienze e Lettere - Libri Scheiwiller, 2007, 2008, 2009.

2 Concepita nel 1838 su iniziativa della Camera di Commercio per «promuovere l'incamminato progresso delle arti e de' mestieri in Milano», vide la partecipazione degli ambienti più sensibili alla crescita produttiva e industriale. La Società di 


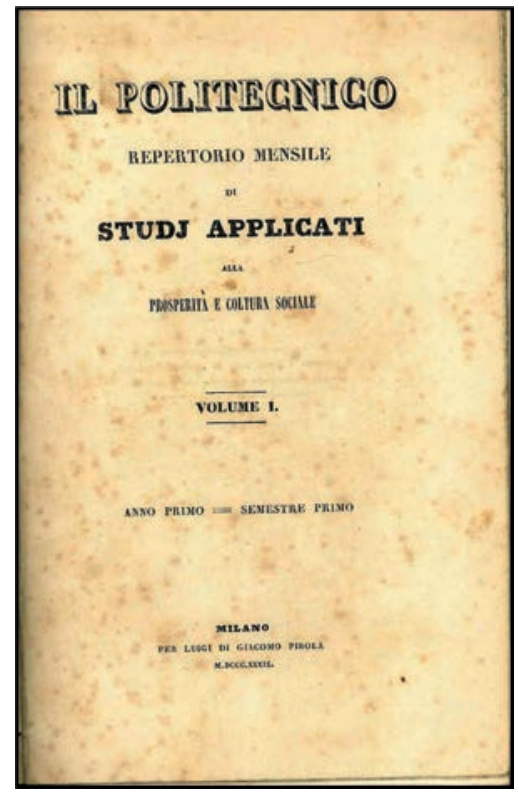

Fig. 2 - Primo numero de «Il Politecnico» (1839).

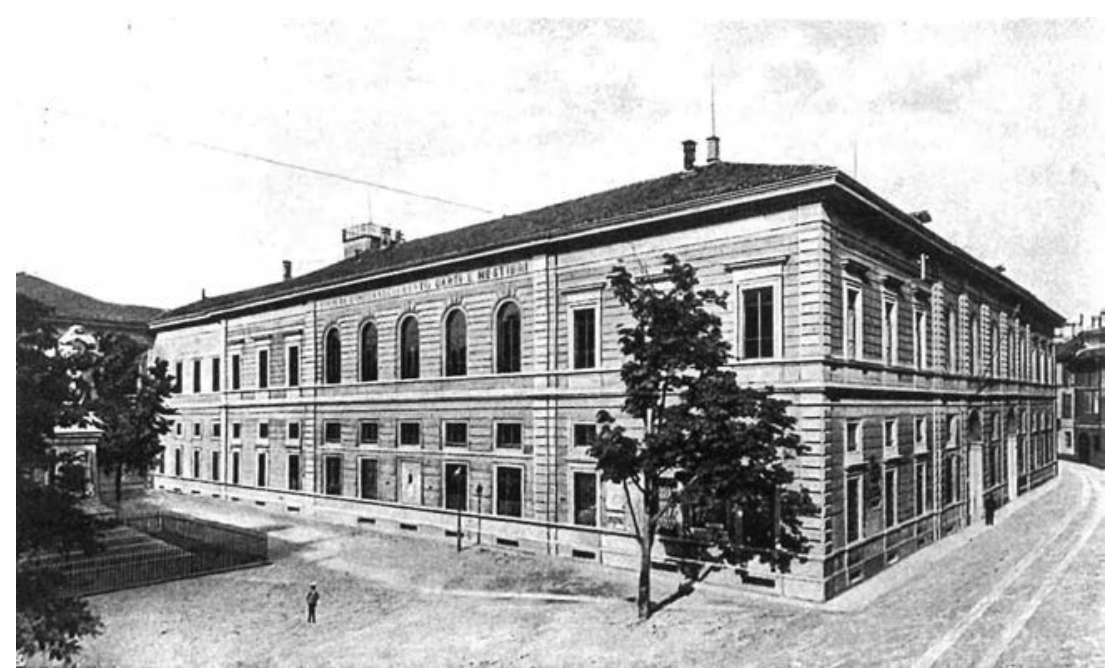

Fig. 3 - La terza sede della Società d'Incoraggiamento d'Arti e Mestieri (1890 - oggi). 
Finita la rivoluzione delle Cinque Giornate, e come tentativo di compensare nei territori dell'Impero la dura politica di centralizzazione con una modernizzazione che migliorasse il benessere economico, si svilupparono molti Progetti e riforme universitarie nel Lombardo-Veneto dopo il 1848. ${ }^{3}$ Essi riguardarono anche la Facoltà matematica e la sua dialettica rispetto a un istituendo Politecnico in Milano, soprattutto auspicato nella riorganizzazione degli studi effimeramente voluta (proprio fino al '59) dal governatore Massimiliano d'Asburgo.

Il documento dell'Istituto Lombardo ${ }^{4}$ «rimane a testimoniare il grado di coscienza civile» di Cattaneo e della parte più avanzata della cultura milanese, anche se non necessariamente «il contenuto di quel rapporto debba essere accolto in blocco». ${ }^{5}$

Certo le sue linee guida resteranno ben presenti in Cattaneo: così come aveva già approfondito certe convinzioni in materia di istruzione documentate negli scritti cronologicamente precedenti raccolti da Ambrosoli, anche nell'esilio luganese non cesserà di ragionare e operare conseguentemente. Mi riferisco, per il passato, al pertinente interesse per l'agricoltura e l'istruzione professionale; ${ }^{6}$ all'attenzione per la cul-

Incoraggiamento sarebbe diventata - con le sue Scuole pratiche, i suoi corsi, i suoi laboratori - un eccellente centro di formazione per operai, operai specializzati, quadri intermedi, quasi un'anticipazione del futuro Politecnico (si veda qui a pag. 167). Cfr. C.G. Lacaita, L'intelligenza produttiva. Imprenditori, tecnici e operai nella Società d'Incoraggiamento d'Arti e Mestieri di Milano (1838-1988), Milano, Electa, 1990.

3 Questo è il titolo del contributo di Alessandra Ferraresi in Le Università e l'Unità d'Italia (1848-1870), nel volume, a cura di A. Ferraresi ed E. Signori, Bologna, Clueb, 2012, tratto dal convegno pavese "Le Università e l'Unità d'Italia" (13-15 giugno 2011).

4 Reso noto per la prima volta - Un grande progetto di riforma scolastica del 1848, redatto da Carlo Cattaneo per incarico dell'Istituto Lombardo di Scienze e Lettere sulla rivista "La Lombardia nel Risorgimento Italiano" (anno XIV, n. 16, luglio 1929) dal suo Direttore Responsabile Antonio Monti1927.

5 Le due citazioni provengono dall'introduzione (Cattaneo educatore) di Luigi Ambrosoli a C. Cattaneo, Scritti sull'educazione e sull'istruzione, Firenze, «Nuova Italia» Editrice, 1963 (p. XVIII): il testo di Cattaneo e della Commissione è intitolato (come nell'articolo di Antonio Monti di cui alla n. precedente, articolo che Ambrosoli naturalmente cita, anche se «costellato» da «numerosi errori di lettura» da lui corretti, p. 77) Sull'ulteriore sviluppo del pubblico insegnamento in Lombardia.

6 Cfr. i due lavori del 1833 e del 1847, ora in C. Cattaneo, Scritti sull'educazione e sull'istruzione, cit. (da adesso in poi Educazione). 
tura tecnica, che si deve anche «fare nel laboratorio», soprattutto in «città che hanno più interessi d'industria»;' sul livello degli studi elementare e ginnasiale in Lombardia; ${ }^{8}$ sulla creazione di nuove cattedre di scienze naturali a Firenze e di un Istituto architettonico a Zurigo.

Così, quando il governo democratico e amico della Repubblica dell'ormai "suo" Canton Ticino chiede a Cattaneo di prospettare una «riforma dell'insegnamento letterario e scientifico nel Ticino» (1852), ${ }^{10}$ Cattaneo riprende i materiali dell'Istituto Lombardo (dai quali stralcia anche alcuni brani) ${ }^{11}$ con la libertà di parlare ora in prima persona, e non manca di insistere sull'importanza dell'istruzione tecnica, importanza sia generale (dovrebbe essere uno dei «fondamenti d'un buon Liceo») sia particolare: per «architetti e ingegneri» dovrebbe valere l'esempio degli «Ingegneri inglesi e americani, i più dei quali non sogliono adire alla laurea universitaria», ${ }^{12}$ e potrebbero essere utili scuole e laboratori come quelli della Società d'Incoraggiamento a Milano.

Veniamo al progetto dell'Istituto Lombardo, e ad alcuni punti qualificanti.

a) A proposito delle scuole elementari, si tratta di dare attuazione al principio governativo (ancora ampiamente disatteso, soprattutto per le «fanciulle») dell'obbligatorietà degli studi per «tutti i fanciulli di ambo i sessi dagli anni sei ai dodici» (Educazione, p. 79). Particolare attenzione va poi riservata alle Scuole infantili, per sordomuti, per ciechi e per carcerati: e in quest'ultimo caso è ben catteneano il pensiero che «il carcere da scuola d'ogni abominio può divenire scuola di onesta operosità» (ib., p. 83).

b) «Per insegnare ai giovani l'esercizio immediato dei mestieri», in par-

7 Nuova scuola di chimica industriale a Milano, 1844, ora in Educazione (la cit. a p. 55).

8 Cfr. i due lavori del 1839, ora in Educazione.

9 Cfr. le due brevi notizie del 1834, ora in Educazione.

10 Sulla riforma dell'insegnamento superiore nel Ticino, ora in Educazione. Che per «insegnamento superiore» qui si intenda quello ginnasiale e liceale è evidente in tutto il discorso, «il cui elemento di maggior rilievo era l'istituzione in Lugano di un liceo cantonale» (Ambrosoli in Educazione, cit., p. 154), effettivamente partito pochi mesi dopo, con Cattaneo professore di filosofia.

11 Cfr. Educazione, pp. 161-162 (è il brano contro le Antologie e le Crestomazie: cfr. il mio successivo, punto c) e pp. 173-174.

12 Educazione, pp. 175 e 177. 
ticolare nell'agricoltura, nelle miniere, nei commerci e «in altri rami delle private aziende e delle pubbliche, come [...] la vigilanza sulle strade ferrate e le macchine a vapore», grande cura deve dedicarsi all'insegnamento tecnico: è per questo che «lo stabilimento, fondato in Milano [nel 1841-'42] dopo lunga aspettazione sotto il nome di Scuola Tecnica, $[\ldots]$ è un gran beneficio alla nostra città. E facciamo voti che codesti Ginnasj commerciali fossero in ognuna di quelle città che hanno un Ginnasio letterario» (ib., pp. 84, 85, 86; corsivi nel testo, qui e nelle cit. seguenti, ove non diversamente precisato).

c) Circa le scuole ginnasiali, sono discussi analiticamente e se del caso criticamente i contenuti e le migliori forme di studio per ogni materia, con insistenza per la lingua italiana e l'esercizio dello scrivere, e per la lettura degli autori diretta e non mediata da Antologie e Crestomazie. Tutte queste riflessioni - lo anticipo qui - non troveranno corrispondenza pour cause nella Legge Casati, dove dei ginnasi (e così pure dei licei) sono forniti elenchi delle discipline caratteristiche, non dissimili da quelle di cui discute Cattaneo, ma senza nessuna problematizzazione.

d) Per i licei, analoga impostazione che per i ginnasi, con una precisazione in più (in linea con la latitudine culturale di Cattaneo): negli studi «si comprendono molte scienze e vastissime e disparate. [...] il Liceo [...] dovrebbe comprendere quelle cognizioni preparatorie bensì alla carriera speciale, ma tali che ogni colto giovane anche in altra carriera deve vergognarsi d'ignorarle», comprese le «scienze esatte e naturali» (ib., p. 99). E l'osservazione che «Tutto ciò che oltrepassa questo limite [e cioè le suddette «cognizioni preparatorie» essenziali per tutti i liceali] deve riservarsi ai corsi speciali di ciascuna carriera, e quindi all'Università» (ib., p. 99) sembra far intendere che l'Università ha come via d'accesso, appunto, i Licei.

e) Infine, riguardo alle Scuole universitarie, ${ }^{13}$ qui interessa soffermarsi sui progetti di riordinamento della Facoltà matematica, dove si for-

13 Solo per l'anticipazione profetica della necessità di una Facoltà medica a Milano (che in effetti avrebbe contribuito a generare una nuova Università, la Statale, nel 1924), ricordo che Cattaneo scrive: «Ma la Facoltà medica, finché la sua sede sia limitata in Pavia non potrà raccogliervi quella moltitudine o varietà di fatti che è materia prima al pratico ammaestramento. Sarebbe quindi mestieri ristabilire le Cliniche, che già fiorivano pochi anni or sono nell'Ospitale di Milano [...], e [...] costituirle in 
mavano (a Pavia ${ }^{14}$ (Fig. 4), per quanto riguarda la Lombardia) gli ingegneri-architetti, con la coda per gli architetti di due anni di Disegno architettonico all'Accademia di Belle Arti di Brera. La relazione di Cattaneo per l'Istituto Lombardo sottolinea però esplicitamente che

La capitale riforma consisterebbe nell'aggiungere agli [studi generali che ora si fanno per tutti gli Ingegneri gli ${ }^{15}$ studj speciali a ciascun ramo dell'arte, applicando il fecondo principio della divisione del lavoro [...] L'ingegnere che governa una strada ferrata e una fabbrica di macchine, non deve avere alla mano quella stessa serie di cognizioni e di dati che serve nella distribuzione di un'acqua irrigatoria o quella che serva alla decorazione di una facciata [...] Il progresso dei tempi vuole che questo fascio di studj disparati si sciolga nelle distinte sue specialità, perché nessuno di essi rimanga senza utile applicazione» (ib., pp. 116-117).

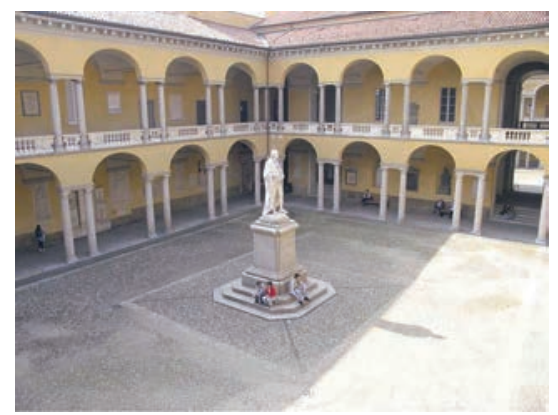

Fig. 4 - Università di Pavia. Cortile del Volta.

Sottolineo, in quanto precede, il rilievo della specializzazione, per rendere significative le applicazioni, secondo quanto richiesto dal «progresso dei tempi»: rilievo consonante con il proposito che porterà avanti accanitamente/pervicacemente e con successo Francesco

una Scuola di Perfezionamento», che dovrebbe «godere quelle stesse condizioni [...] che godono le Cliniche Universitarie a Pavia» (Educazione, pp. 109-110).

14 Cfr. V. Cantoni e A. Ferraresi (a cura di), Ingegneri a Pavia tra formazione e professione. Per una storia della Facoltà di Ingegneria nel quarantesimo della rifondazione, Milano, Cisalpino, 2007.

15 In Educazione, p. 116, è saltato il tratto tra quadre, che invece era pertinentemente presente nella trascrizione di Antonio Monti (cfr. nn. 4 e 5). 
Brioschi 1857 (Fig. 5), con le specializzazioni del triennio d'applicazione del suo Politecnico. Non amato da Cattaneo anche perché era partecipe dell'elaborazione della Legge Casati, Brioschi negli anni '50 aveva cercato invano - per divergenze politiche con Cattaneo - di far rinascere «Il Politecnico» dopo la prima serie e finirà davvero nel 1865 per acquisire (chiusa la seconda serie cattaneana) la rivista, dirigendola e modificandone in profondità la fisionomia culturale. ${ }^{16}$ Circa la Legge Casati, Brioschi l'avrebbe forzata - per così dire - subito alla fondazione della sua Scuola (1863), per istituire un percorso universitario con una dipendenza dalle Facoltà matematiche dell'Università ridotta ai soli primi due anni su tre, infine (1875) per acquisire anche il biennio preparatorio dentro il Politecnico e autonomo dall'Università. A proposito dell'utilità dell'«applicazione», Cattaneo aggiunge: «Ritenuto adunque comune a tutti gl'Ingegneri l'attuale studio universitario, converrebbe sovrapporvi un secondo ordine di studj pratici e di perfezionamento» (ib., p. 117), intravvedendo una scuola politecnica (le «distinte specialità» dell'ingegnere) che completi in senso pratico la formazione teorica dell'Università.

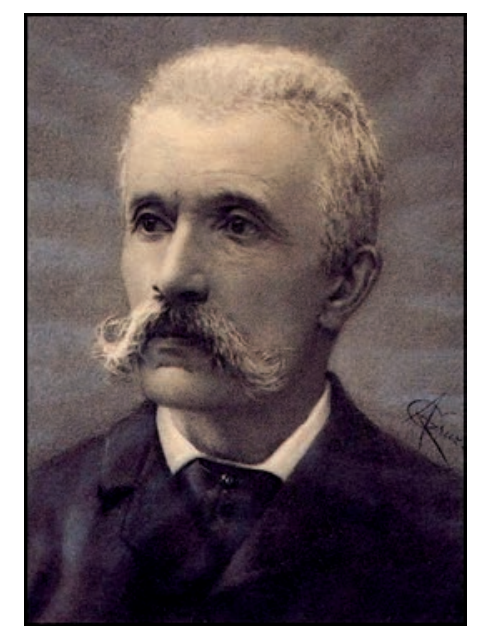

Fig. 5 - Francesco Brioschi (1824-1897).

16 Cfr. A. Galbani e A. Silvestri (a cura di), Da "Il Politecnico" di Cattaneo al Politecnico di Brioschi, Politecnico di Milano, 2003. 
«Ove dovrebbero istituirsi questi nuovi studj» (ib., p. 120) Cattaneo qui lo dice solo allusivamente. Infatti non c'è il nome di una città, ma si ipotizza «di deputare alle lezioni [...] lo stesso Ufficio delle pubbliche costruzioni», a Milano ${ }^{17}$ aggiungo io, con esercitazioni e dimostrazioni «tanto sui progetti giacenti presso la Direzione stessa, quanto $^{18}$ sui modelli e sulle macchine del Gabinetto Tecnologico dell'Istituto», e cioè l'Istituto Lombardo pure a Milano. Milano è citata esplicitamente più tardi da Cattaneo stesso, ${ }^{19}$ reso più polemico nel contrastare una legge del governo sabaudo, perdipiù nominata dal nome di uno dei pochissimi ministri milanesi in quello stesso governo, Gabrio Casati (Fig. 6), esponente di punta dell'aristocrazia moderata e filo-piemontese ai tempi delle Cinque Giornate: «già in quel progetto di riforma» dell'Istituto Lombardo nel 1848, «benché redatto non senza pericolo, si toccò pure [...] di una scôla politecnica civile e militare. Essa doveva costituirsi in Milano». E anche poco dopo, nella seconda lettera pubblica al Senatore Carlo Matteucci 1844 pubblicata sul «Politecnico» nel 1862, è precisato che l'importantissima (per Cattaneo) «facultà d'Ingegneri agronomi» ${ }^{20}$ della quale fornisce un articolato piano di studi - con il controcanto della polemica sulla Legge Casati -, va «trasferita da Pavia in Milano». ${ }^{21}$ Così «la facultà di Pavia, sfrondata di questo estraneo ramo, $[. .$.$] diviene tanto più veramente \mathrm{e}$ più specificatamente matematica», realizzando anzi una «Scôla Normale pei professori»: sarebbe stato dell'anno successivo, a Milano, l'avvio delle scuole normali per i futuri insegnanti secondari (cfr. qui alle pp. 178-179) di discipline scientifiche (la sezione appunto normale del

17 Nello stesso 1805 della proclamazione del Regno d'Italia, sull'esempio francese e per favorire la politica dei lavori pubblici, fu istituita a Milano la Direzione generale di acque e strade; nel 1818 fu adottata la denominazione Direzione lombarda (e, a Venezia, veneta) della pubbliche costruzioni, e nel 1859 - poco prima della Legge Casati - quella di Corpo del Genio civile.

18 Anche qui mi sono attenuto alla trascrizione di Antonio Monti (cfr. nn. 4, 5 , 15), mentre Educazione (p. 120) porta «tanto [...] quando».

19 La nuova legge del pubblico insegnamento, 1860, ora in Educazione. La citazione che segue è a p. 198 .

20 Di alcuni rami d'insegnamento scientifico da istituirsi in Milano. Altra lettera di Carlo Cattaneo al senatore Matteucci ministro della Istruzione Publica, in Educazione, p. 227.

$21 \quad I b .$, p. 229. 
Politecnico) e di discipline letterarie (la coeva Accademia scientificoletteraria).

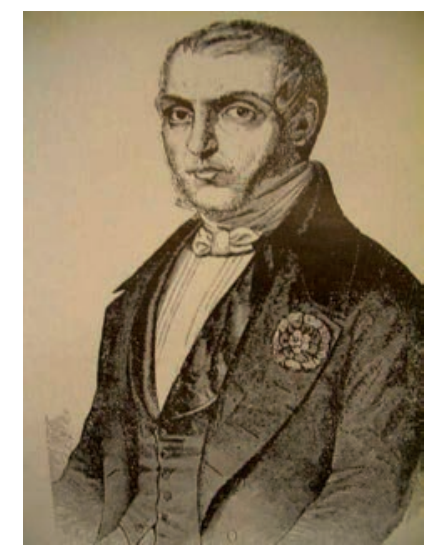

Fig. 6 - Gabrio Casati (1798-1873)

Circa l'impostazione «civile e militare» che manca al progetto scolastico della Legge Casati, l'accusa di Cattaneo è di aver dimenticato la funzione educativa della scuola in vista della difesa della patria, escludendo addirittura l'istruzione militare dalle competenze del Ministero della Pubblica Istruzione (dal quale dipendono infatti, secondo l'art. 3 della Legge, «tutte le scuole e gli istituti d'istruzione e d'educazione, e rispettivi stabilimenti», ${ }^{22}$ «eccettuati gli istituti militari e di nautica»). Un'altra critica riguarda l'agricoltura, «non solo per difetto d'istituti agrari [...]; ma perché gli ingegneri [...] non ricevono nel loro corso di studj nemmeno uno spruzzo di scienza agraria» (Educazione, p. 192). Quanto all'industria, si ricorda l'esempio della Scuola d'Incoraggiamento («immedesimandosi [...] sempre più quell'istituzione colla tendenza del Politecnico»), dei suoi uomini, dei suoi corsi, dei suoi laboratori (per es. di chimica, fisica industriale, setificio, locomotive), per concludere: «Ma

22 Si noti: stabilimenti, come li chiamava Cattaneo nel '48 (cfr. qui a p. 163). Ma in quei tempi era corrente l'originario significato del vocabolo: «organizzazione [...] di uno scopo pubblico o di un interesse sociale», prima che «fabbricato in cui l'organizzazione svolge la sua attività» (Grande dizionario della lingua italiana di Salvatore Battaglia, Torino, UTET, 2000, ad vocem). 
devono dunque le Camere di Commercio e le società private fornire i professori di alte scienze alle vostre università?» (ib., p. 197). Tornando al progetto dell'Istituto Lombardo «prima dell'eruzione del 1848», scrive sempre Cattaneo, «dato un rapido sguardo alla nuova legge, non ci parve nemmen ravvisarvi alcuna delle molte proposte di allora» (ib., p. 198).

Cattaneo non sa che, di lì a pochissimi anni, l'inviso Brioschi avrebbe lottato (nella preparazione della Legge Casati) contro l'ipotesi di una sola Scuola di Applicazione per gli Ingegneri a Torino, anche perché «Nelle pianure lombarde, egli dice, hanno assai maggiore importanza le conoscenze di agricoltura e di idraulica». ${ }^{23}$ E l'Istituto Tecnico Superiore che avrebbe fondato a Milano sarebbe stato radicato profondamente nella continuità e nella condivisione di professori, gabinetti e laboratori con la Società d'Incoraggiamento, e dotato innovativamente di una sezione meccanica (poi industriale) accanto a quella tradizionale civile, essendo inoltre presente nella sezione civile un corso intitolato all'Idraulica fluviale e agricola, e un altro all'Agronomia, avendo anzi Brioschi fatto trasferire da Pavia su questa cattedra Carlo Pasi1857, ultimo esponente della scuola pavese di agraria e primo al Politecnico.

La continuità del pensiero di Cattaneo non cesserà di dare frutti. A favore delle specializzazioni egli si era già espresso, sempre nel 1862 e sempre sulla seconda serie del «Politecnico», nella prima «lettera al Senatore Matteucci»: Sul riordinamento degli studj scientifici. Cattaneo era stato richiesto da Matteucci, uno studioso che Cattaneo apprezzava e che era stato collaboratore della sua rivista, di un parere sul suo progetto di riforma dell'istruzione superiore poi non approvato.

È l'occasione per ribadire la necessità delle specializzazioni nell'ingegneria (ma discorso analogo è argomentato per il diritto e la medicina): ${ }^{24}$ «voi darete all'Italia ingegneri architetti, ingegneri idraulici, ingegneri agronomi, ingegneri censuarj, ingegneri delle miniere, ingegneri militari, navali, geografi, ferroviari, e uomini nati col genio mecanico»

23 Cfr. in M. Moretti e I. Porciani, Da un frammento a un testo. Estate 1859, la discussione preparatoria sulla Legge Casati, in Le Università e l'Unità d'Italia (18481870), cit., pp. 33-34, uno stralcio della Relazione al ministro Casati della Commissione preparatoria della legge con la verbalizzazione di un intervento di Brioschi.

24 «Alle scienze mediche naturalmente offronsi più grandi occasioni nelle più grandi città [che] nelle piccole città universitarie»(Educazione, p. 215): anche qui Cattaneo sembra alludere (cfr. n. 13) a Milano versus Pavia. 
(Educazione, p. 208). Ma «la suddivisione [, la] specificazione» (ib.) devono adattarsi alle tradizioni, alle competenze, alle necessità dei singoli territori: in Lombardia «una classe [...], alla quale poco veramente necessita sapere in che la trabeazione dorica si distingua dalla ionica», viene istruita con «una solida e larga preparazione scientifica d'ingegneri-agronomi, ingegneri-silvicultori, piuttosto che d'ingegneri-architetti» (ib., $\mathrm{p}$. $205) ;_{2}^{25}$ «voi potreste nella facultà speciale per gli ingegneri toscani e sardi introdurre studio normale di drenatura e marnatura, [con] nozioni geologiche, almeno per la parte della stratigrafia» (ib., pp. 206-207); «nelle facultà di Bologna e Padova [...] sono di somma opportunità le scienze idrauliche e sopratutto la fisica de' fiumi» (ib., p. 207); sono da prevedere da un lato «la scôla degli ingegneri navali nel più gran centro di costruzione, o almeno di navigazione», e dall'altro «gli ingegneri delle miniere in luogo dove non possono girare li occhi senza vedersi affacciare da monti e valli le evoluzioni del globo. E gli ingegneri architetti, davvero architetti e ministri del bello, non avranno a sfogliar volumi per raccogliervi una fioca ombra dei monumenti di Roma» (ib., p. 209). La lettera si chiude con l'invito a insegnare in ogni facoltà le «scienze militari» (ib., p. 224), anziché «l'istruzione teologica» che proviene da un'«autorità ecclesiastica [...] che cinta d'armi straniere combatte l'Italia in Roma» (ib., p. 222).

\section{LA LEGGE CASATI}

Al termine della seconda guerra di indipendenza, com'è noto, la Lombardia fino al Mincio era ceduta dall'impero asburgico alla Francia di Napoleone III, che l'avrebbe consegnata a Vittorio Emanuele II. L'annessione di fatto della Lombardia al Piemonte fece avvertire (tempestivamente e in anticipo rispetto all'Unità d'Italia) l'esigenza di un'unificazione dei relativi modelli formativi.

La Legge Casati ${ }^{26}$ fu una legge quadro per l'intera istruzione: quel-

25 Di questa ipotizzata facoltà di ingegneri agronomi Cattaneo discuterà nel dettaglio - fino alla proposta di una sorta di piano degli studi - nella cit. seconda lettera al Senatore Matteucci (per cui cfr. qui p. 166 e relativa nota 20).

26 Cfr. su Gabrio Casati la voce di Luigi Ambrosoli, in Dizionario Biografico degli Italiani, vol. 21, 1978, ad vocem. 
la primaria con la novità sorprendente benché ancora di principio, in un'Italia eccezionalmente poco alfabetizzata, di una cultura basica obbligatoria; e anche l'istruzione secondaria, ${ }^{27}$ e quella superiore, come vedremo, profondamente innovate, tenendo dietro alla modernizzazione tecnica avanzante. La Legge Casati, nella sua progressiva estensione a tutto il Paese, avrebbe operato su un panorama preunitario assolutamente eterogeneo, essendo soggetta a critiche ${ }^{28}$ sia di parte progressista sia di parte moderata, ma non se ne può negare la complessità e il rilievo, in particolare nel settore tecnico-scientifico.

Concepita da una commissione inizialmente con membri solo torinesi (tra i quali Quintino Sella $1861^{29}$ (Fig. 7), appena nominato da Casati membro del Consiglio superiore dell'Istruzione), la Legge Casati ${ }^{30} \mathrm{fu}$ poi aperta al contributo lombardo e di altri Stati preunitari settentrionali: e proprio in questo contesto stava per manifestarsi la forte personalità di Francesco Brioschi, ${ }^{31}$ allora professore dell'unico ateneo della Lombardia, Pavia, ma presto fondatore del Politecnico di Milano.

Le due grandi novità della Legge Casati che vorrei adesso focalizzare sono da un lato (paragrafo 4) la nascita dei cosiddetti "stabilimenti tecnici", ${ }^{2}$ cioè le Scuole tecniche post-elementari e gli Istituti tecnici secondari; dall'altro (paragrafo 5) la creazione delle due prime Scuole superiori per gli ingegneri proprio a Torino e a Milano, e a Milano inoltre un primo nucleo via via sempre più significativo, l'Accademia scien-

27 Su cui si veda ora L'istruzione secondaria nell'Italia unita (1861-1901), a cura di C.G. Lacaita e M. Fugazza, Milano, Franco Angeli, 2013.

28 Per una rassegna a volo d'uccello delle valutazioni negative e positive della storiografia sulla Legge Casati, cfr. G. Decollanz, Storia della scuola e delle istituzioni educative, Bari, Laterza, 2005, pp. 5-16.

29 Cfr. il volume Quintino Sella scienziato e statista per l'Unità d'Italia, Roma, Scienze e Lettere, 2013 (che proviene dall'omonimo Convegno - promosso dai Lincei e dalla Fondazione Sella - tenuto a Roma, 5-6 dicembre 2011).

30 Su cui si veda ora M. Moretti e I. Porciani, Da un frammento a un testo. Estate 1859, la discussione preparatoria sulla Legge Casati, in Le Università e l'Unità d'Italia (1848-1870), cit.

31 Cfr. Francesco Brioschi e il suo tempo (1824-1897), 3 voll. (I Saggi, II Inventari, III Scritti e discorsi), progetto editoriale di C.G. Lacaita e A. Silvestri, Milano, Franco Angeli, 2000, 2000, 2003.

32 Cfr. A. Silvestri, Francesco Brioschi e l'istruzione tecnica, in L'istruzione secondaria nell'Italia unita (1861-1901), cit. 
tifico-letteraria, ${ }^{33}$ di quella che sarebbe stata la Facoltà di Lettere e filosofia alla nascita nel 1924 dell'Università degli Studi di Milano, per iniziativa soprattutto di un altro transfuga da Pavia, il ginecologo Luigi Mangiagalli 1901 .

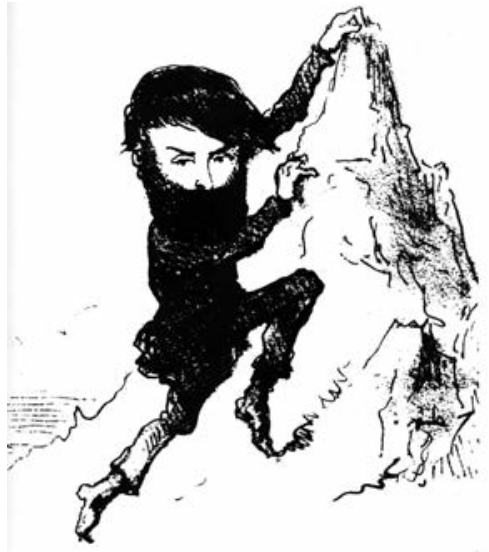

Quintino Sella è andato a prendere d'assalto il gran Monviso...

Fig. 7 - Una caricatura di Quintino Sella (1827-1884).

\section{Nella Legge Casati, GLi "STABILIMENTI TeCNiCi"}

Nel Lombardo-Veneto già nel 1838 l'imperial-regio governo aveva emanato un "Regolamento per le Scuole tecniche di Milano e di Venezia", che in realtà si aprirono più tardi. A Milano, nel 1841-'42 nasce la Scuola tecnica o Scuola reale, quella che assumerà, nel 1884, la denominazione attuale di Istituto Carlo Cattaneo (Fig. 8). Nel 1842-'43 partiva la Scuola tecnica di Venezia in Campo San Procolo. Mentre a Torino, nel 1848 una scuola tecnica "sperimentale" e nel 1856 una

33 Cfr. il vol. G. Barbarisi, E. Decleva, S. Morgana (a cura di), Milano e l'Accademia scientifico-letteraria. Studi in onore di Maurizio Vitale, 2 tomi, Milano, Cisalpino, 2001, e in particolare - nel I tomo - E. Decleva, Una facoltà filosoficoletteraria nella città industriale. Alla ricerca di un'identità (1861-1881). 
scuola industriale non ebbero successo per l'«ancora incerta vocazione industriale della città». ${ }^{34} \mathrm{E}$ al 1853 risale la fondazione da parte di Leopoldo II dell'Istituto tecnico toscano.

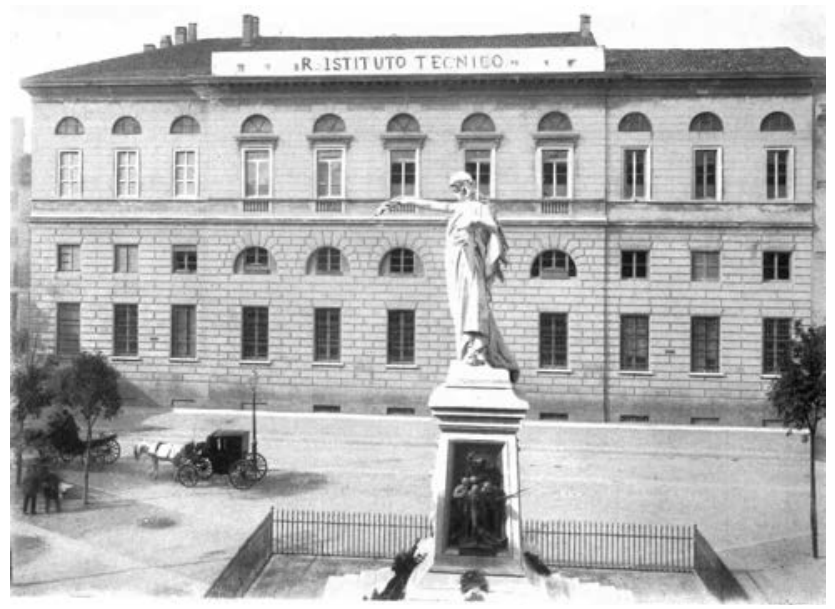

Fig. 8 - La facciata su piazza Mentana del Regio Istituto Tecnico, poi intitolato a Carlo Cattaneo.

La Legge Casati, a proposito degli stabilimenti tecnici, riporta un'affermazione programmatica che riassume la sensibilità maturata, anche in un Paese arretrato come il nostro, a séguito della prima rivoluzione industriale:

L'istruzione tecnica ha per fine di dare ai giovani che intendono dedicarsi a determinate carriere del pubblico servizio, alle industrie, ai commerci ed alla condotta delle cose agrarie, la conveniente cultura generale e speciale (art. 272).

Questo riconoscimento, che l'innovazione necessita di tecnici dotati di conoscenze teoriche e applicative in grado di giovare alla moderna imprenditoria, consuona abbastanza con l'auspicio di Cattaneo nel '48 a «insegnare ai giovani l'esercizio immediato dei mestieri, [...] a quei mol-

34 Cfr. E. De Fort, L'istruzione primaria e secondaria e le scuole tecnico-professionali, in Storia di Torino. VI. La città nel Risorgimento (1798-1864), Torino, Einaudi, 2000 . 
tissimi [...] destinati [...] agl'impieghi d'ordine, alle arti, alle aziende commerciali» (Educazione, pp. 84 e 87). ${ }^{35}$ Né la precisazione della Legge Casati, che gli «Istituti Tecnici potranno essere aperti, a misura che il bisogno se ne farà sentire, nelle città che sono centro di un più notevole movimento industriale e commerciale» (art. 283), è dissimile dall'insistenza di Cattaneo poiché sul territorio le scuole commerciali o tecniche «s'adattino alle circostanze d'ogni provincia, come per es. le Scuole di Setificio istituite dalla Società d'Arti e Mestieri si adattano alle particolari circostanze di Milano e di Como» (Educazione, p. 87).

La Legge Casati prevede un'articolazione degli studi tecnici (come ho già accennato) in due gradi entrambi di tre anni, le Scuole tecniche e gli Istituti tecnici. Le Scuole tecniche sono successive alle Scuole elementari, comunali e gratuite, distinte anche queste in due gradi, inferiore e superiore, ciascuna di due anni, il primo obbligatorio (art. 326): ${ }^{36}$ con la minaccia che siano «puniti a norma delle leggi penali dello Stato» le famiglie che non provvedono o anzi consentono che siano «impiegati $[\ldots]$ fanciulli che siano in età di frequentare la scuola pubblica» (art. 327). Anche Cattaneo continua a ribadire l'importanza e l'inadeguatezza degli studi elementari, fino all'ultimo dei suoi contributi sul «Politecnico» riuniti in Educazione. Per esempio, in Raccolta di alcune proposte di leggi e di varj scritti sulla pubblica istruzione del Senatore C. Matteucci (1865), Cattaneo afferma: «Tutti i paesi avvertono la meravigliosa potenza che si cela nelle scôle elementari, che, più delle secondarie e delle universitarie, formano la cultura generale e il carattere della nazione» (ib., p. 241); «Il Parlamento [...] rivolga esso all'istruzione elementare una parte di quel denaro che disperde nelle troppe università, nelle tante academie di belle arti» (ib., p. 247).

Secondo la Legge Casati, le Scuole tecniche sostanzialmente approfondiscono i rudimenti - «la facoltà di leggere, scrivere e conteg-

35 Così come, almeno in via di principio, non è evidentemente estranea alla Legge Casati la «condotta delle cose agrarie», che stava così a cuore a Cattaneo. Cfr. inoltre, qui, la n. 25.

36 Cattaneo da parte sua ricordava l'obbligo, solo teorico come ho già accennato, che nel Lombardo-Veneto «dovessero frequentare tutti i fanciulli di ambo i sessi dagli anni sei ai dodici» le scuole elementari, ripartite in "minori" e "maggiori" (Educazione, pp. 79 e 87). 
giare», aveva scritto Cattaneo nel '48 (Educazione, p. 79) - forniti dalle elementari; mentre il secondo ciclo, gli Istituti tecnici, inserisce nuove materie applicative come il diritto amministrativo e commerciale, l'economia, le scienze sociali inclusa la statistica, la chimica, la fisica e la meccanica elementare, il disegno, senza dimenticare «Agronomia, e storia naturale» (art. 275) di cattaneana memoria.

È poi indicativo della valorizzazione di questi nuovi studi che le Scuole e gli Istituti tecnici siano equiparati agli «stabilimenti di istruzione secondaria classica» ${ }^{37}$ (art. 285), e cioè il ginnasio (cinque anni) più il liceo (tre anni). Invece non è ancora esplicitamente prevista - al contrario che nella legislazione asburgica, e anche su questa lacuna si appunteranno molte critiche lombarde ma non solo (come ho appena osservato alla n. 37) - la possibilità per i licenziati degli Istituti tecnici di iscriversi all'Università. Ben presto un Regio Decreto del 19 settembre 1860 (n. 4315) istituisce la sezione fisico-matematica degli Istituti tecnici, che consente l'iscrizione alle Facoltà di scienze matematiche, fisiche e naturali delle Università del Regno e quindi - nelle forme che vedremo alle pp. 175-177, diversificate tra Torino e Milano - alle Scuole di applicazione per gli ingegneri dei due Politecnici creati dalla Legge Casati stessa.

Da tale sezione fisico-matematica sarebbe uscita una parte non trascurabile delle future classi dirigenti dell'Italia unita. Cito almeno per Milano i nomi di industriali di chiara fama anche internazionale come Giovanni Battista Pirelli, Alberto Riva e Franco Tosi; architetti, restauratori e uomini delle istituzioni come Luca Beltrami 1895 o Piero Portaluppi1960; oppure, in epoca fascista, il rettore Gaudenzio Fantoli 1906 e il professore-ministro Giuseppe Belluzzo1922; o capitani d'industria quali Giacinto Motta1906 alla Edison, Giuseppe Cenzato alla Società Meridionale di Elettricità; oppure Ercole Bottani1940, professore di Elettrotecnica al Politecnico e fondatore del CESI (Centro

37 Ricordo che Cattaneo auspicava che la Scuola Tecnica milanese fosse modello per «Ginnasj commerciali o industriali» da istituire «in ognuna di quelle città che hanno un Ginnasio letterario» (Educazione, p. 87). Mentre tra le critiche alla Legge Casati, Mauro Macchi lamentava la preponderanza delle scuole classiche rispetto alle tecniche, e Stanislao Cannizzaro era contrario alla mancata equivalenza - ai fini dell' $i$ scrizione alle Facoltà matematiche - dei due profili di licenza (cfr. C.G. Lacaita, La svolta unitaria negli studi superiori, in Le Università e l'Unità d'Italia (1848-1870), cit., p. 43). 
Elettrotecnico Sperimentale Italiano Giacinto Motta) e della Metropolitana milanese..$^{38}$

\section{La Legge Casati e L'istruZione tecnica superiore a TORINO E A Milano}

Veniamo ai due Politecnici storici. Dell'uno - la Scuola di applicazione per gli Ingegneri di Torino, capitale del regno sabaudo e tra poco d'Italia - nella Legge Casati si legge: «Alla Facoltà di Scienze Fisiche e Matematiche dell'Università di Torino sarà annessa una Scuola di applicazione [...] per gli Ingegneri» (art. 53). La Scuola (Fig. 9) parte subito (1860) e accoglie i laureati della Facoltà matematica (tre anni) per due anni di applicazione: l'obiettivo è quello di un'alta cultura di base fisicomatematica, secondo il modello dell'École Polytechnique francese. L'ingegnere che vi viene formato è sostanzialmente quello civile, mentre l'ingegneria industriale a Torino sarà avviata più tardi, appoggiandosi anche e non marginalmente al Museo industriale (1862), quando alle sue primitive funzioni espositive si sovrapposero, via via prevalendo, quelle didattiche. (Sarà alla fusione delle due suddette istituzioni torinesi, 1906, che nascerà ufficialmente, con questo nome, il Politecnico di Torino).

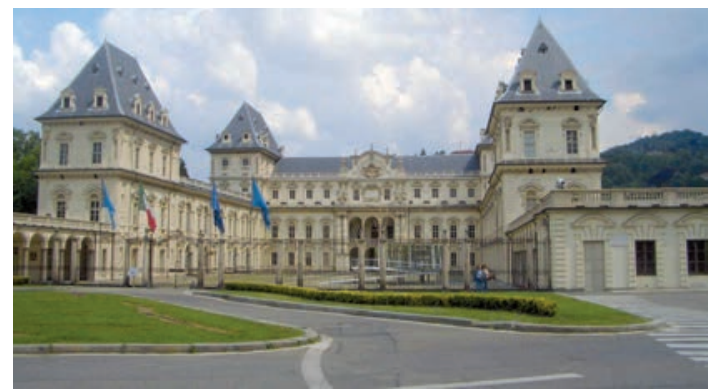

Fig. 9 - Il Castello del Valentino, già sede della Regia Scuola di Applicazione per gli ingegneri, ora sede della Facoltà di Architettura dell'Università di Torino.

38 Questi e altri esempi, con i relativi riferimenti bibliografici, sono presentati nel mio contributo La cultura politecnica e l'industria, nel volume (da un convegno dell'Accademia dei Lincei e della Fondazione Edison) A. Quadrio Curzio1988 e M. Fortis (a cura di), L'industria nei 150 anni dell'Italia unita. Paradigmi e protagonisti, Bologna, Il Mulino, 2012, pp. 103-129. 
Milano, invece, non più capitale di un regno, e con un tessuto della sua cultura tecnica e imprenditoriale così evoluto da richiedere un delicato coordinamento di forze potenzialmente sinergiche, è evocata nella Legge Casati a proposito di un R. Istituto tecnico superiore da precisarsi con un futuro Decreto applicativo (art. 310). Il fondatore Brioschi progressivamente acquisisce rilievo a Torino nel Ministero della Pubblica Istruzione, vi avvia una proficua collaborazione con Quintino Sella, ${ }^{39}$ e riesce a smuovere le lentezze centrali; mentre a Milano sa raccordare nel nuovo Politecnico tutte le istituzioni pertinenti: la Società d'Incoraggiamento d'Arti e Mestieri con le sue Scuole, con i suoi ricchi laboratori, con i suoi professori (lo stesso Brioschi, e soprattutto il cofondatore del Politecnico ${ }^{40}$ Giuseppe Colombo1862); il Comune per l'utilizzo di personale e strutture del Museo civico di storia naturale (che era stato fondato nel 1838 proprio dal podestà Gabrio Casati prima delle Cinque Giornate di Milano e prima del suo esilio torinese); e a Brera (Fig. 10): l'Istituto Lombardo e il relativo gabinetto tecnologico (Fig. 11), l'Osservatorio astronomico (con le proprie collezioni e con la docenza di suoi uomini di punta, per esempio Giovanni Schiaparelli 1862 e poi Giovanni Celoria1873), l'Orto botanico per i corsi di agronomia ${ }^{41}$ e per la sezione "normale" del Politecnico, e il Gabinetto numismatico per gli insegnamenti archivistici dell'Accademia scientifico-letteraria.

Circa la struttura, il Politecnico di Milano (Fig. 12) è subito proteso verso la specializzazione, sul modello delle Scuole di ingegneria di cultura tedesca, compreso il Politecnico di Zurigo (modello caro a Brioschi, che aveva conosciute di persona le principali realtà politecniche europee in un famoso viaggio di studio del 1858 in Francia, Svizzera, Germania); la nuova scuola comprende solo (per ora) il triennio d'applicazione, mentre a monte la formazione di base matematica, fisica, ecc. doveva essere acquisita non autonomamente al Politecnico,

39 Nonostante le reciproche idee sul Politecnico di Milano fossero inizialmente non coincidenti: cfr. il mio lavoro Il contributo [di Sella] all'avvio del Regio Istituto Tecnico Superiore di Milano, nel volume Quintino Sella scienziato e statista per l'Unità d'Italia, cit.

40 Cfr. G. Colombo, Industria e politica nella storia d'Italia. Scritti scelti: 18611916, a cura di C.G. Lacaita, Roma-Bari, Cariplo-Laterza, 1985.

41 Si vedano invece le critiche alla Legge Casati di Cattaneo, che vi riteneva «negletta l'agricoltura», Educazione, p. 192: ne parlavo in 2.4. 
ma nei soli primi due anni di una Facoltà matematica in qualsiasi università del Regno (gli allievi del Politecnico di Milano, dunque, al contrario che a Torino, non erano preliminarmente "laureati" in matematica, e se lo erano potevano iscriversi a Milano al second'anno della Scuola d'applicazione).

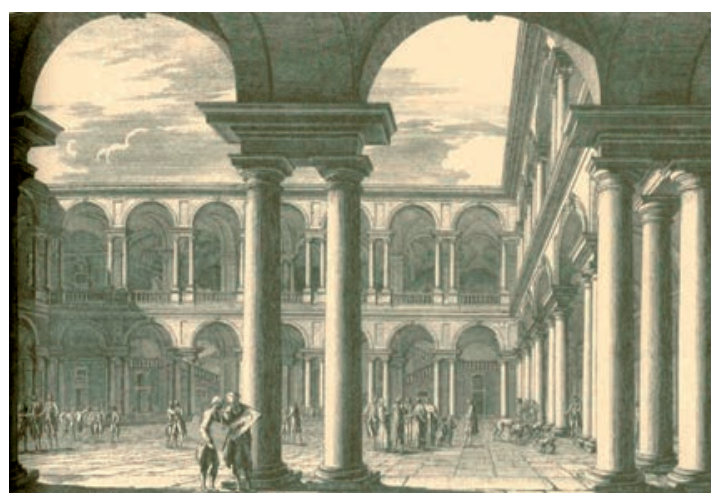

Fig. 10 - Cortile del Palazzo di Brera. Incisione di Domenico Aspari (1786).

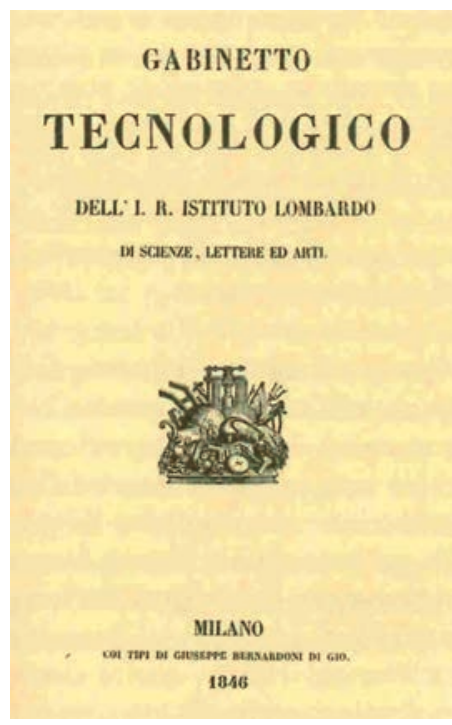

Fig. 11 - Frontespizio del catalogo del Gabinetto Tecnologico (1846). 


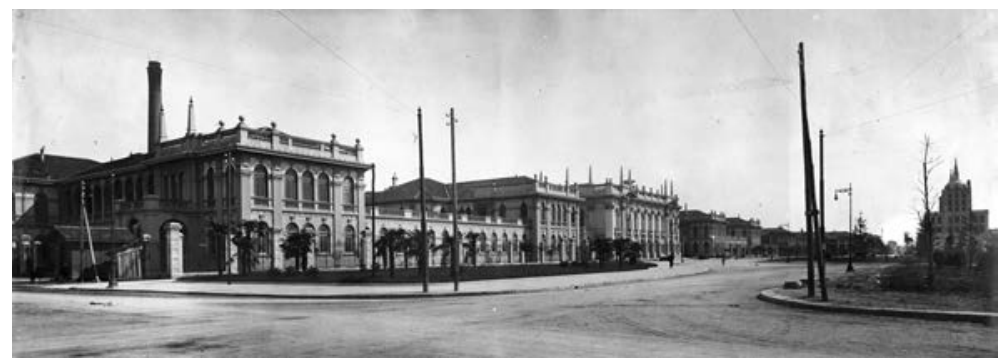

Fig. 12 - Politecnico di Milano. Sede di piazza Leonardo da Vinci (1927).

Quest'insistenza per la specializzazione a Milano non sottovalutava l'importanza delle discipline del biennio preparatorio, e non poteva essere diversamente da parte di un matematico di chiara fama come Brioschi: tant'è vero che Brioschi, caparbiamente, autorevolmente (data la sua posizione di Segretario generale al ministero di Torino, data la presenza nel Consiglio Superiore della Pubblica Istruzione, e poi nel ruolo di Senatore onnipresente in qualsiasi decisione riguardasse l'Università e non soltanto), nel 1875 riuscirà a ottenere - con il sostegno economico del Comune e della Provincia di Milano - la Scuola preparatoria del Politecnico autonoma dalle Università, con una soluzione che tarderà ad arrivare a Torino stessa e nelle altre Scuole di ingegneria, nate perlopiù da Facoltà matematiche, dopo l'esportazione nell'Italia unita del modello della Legge Casati.

Il Politecnico di Milano parte subito non come scuola generalista, ma con più sezioni: l'ingegneria civile era quella in definitiva sottratta più $\mathrm{o}$ meno parzialmente a Pavia ${ }^{42}$ ma nuovissima è la sezione degli ingegneri meccanici, fortemente voluta da Colombo (Fig. 13), e che dopo qualche anno (1873, a cavallo proprio della grande inchiesta industriale) ${ }^{43}$ avrebbe allargato il tiro diventando sezione industriale, per estendere il nome ai progressi della chimica e alle nascenti applicazioni industriali dell'elettricità. ${ }^{44} \mathrm{La}$ terza sezione, la scuola "normale"

42 Cfr. Ingegneri a Pavia tra formazione e professione. Per una storia della Facoltà di Ingegneria nel quarantesimo della rifondazione, cit.

43 Cfr. C.G. Lacaita, Il Politecnico di Milano, in Il Politecnico di Milano 1863. 1914, Milano, Electa, 1981, p. 16.

44 Cfr. A. Silvestri, I nuovi saperi dell'ingegneria al Politecnico di Milano, in Le Università e l'Unità d'Italia (1848-1870), cit. 
per formare i futuri docenti ${ }^{45}$ di discipline scientifiche per le scuole di istruzione secondaria, è il pendant tecnico della già ricordata (altro cruccio pavese!) Accademia scientifico-letteraria, ${ }^{46}$ la futura (1924) Facoltà di Lettere della Statale di Milano.

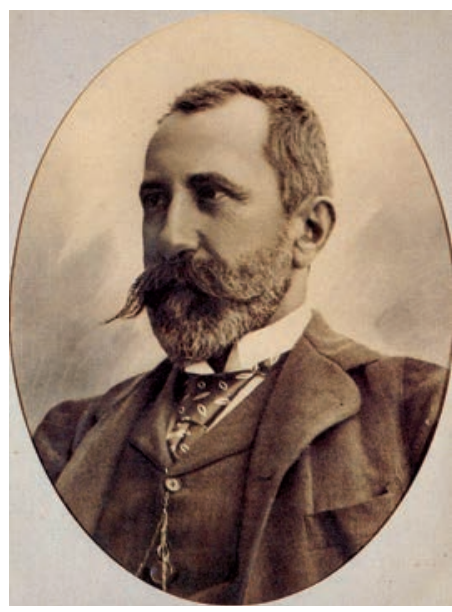

Fig. 13 - Giuseppe Colombo (1836-1921).

Subito a due anni dall'apertura, e cioè nel 1865, come promesso da Brioschi nel discorso inaugurale, è aggiunta la sezione speciale di architettura civile, con la determinante sinergia dell'Accademia di belle arti di Brera, in particolare di Camillo Boito1893 (Fig. 14): tuttavia non veniva ancora soppresso il diploma biennale di Disegno architettonico dell'Accademia,${ }^{47}$ attento agli aspetti piuttosto artistici che tecnicocostruttivi, e le iscrizioni degli architetti civili al Politecnico nei primi decenni non sarebbero state numerose (tra l'altro per la non chiara distinzione rispetto agli ingegneri civili).

45 Anche Cattaneo, nel progetto dell'Istituto Lombardo, prevedeva l'opportunità di «scuole destinate a preparare i Professori», poiché «in generale chi insegna dovrebbe aver percorso non solo l'ordine di studj che professa, ma un grado superiore»: e tale «Facoltà Professoria [dovrebbe] collocarsi in quella città dove naturalmente maggiore fosse il concorso degli uditori», ancora Milano? (Educazione, rispettivamente alle pp. 122, 123, 124).

46 In merito alla nascita e all'evoluzione dell'Accademia scientifico-letteraria, cfr. Milano e l'Accademia scientifico-letteraria. Studi in onore di Maurizio Vitale, cit.

47 Che era menzionato dallo stesso Cattaneo in questa frasetta en passant: 


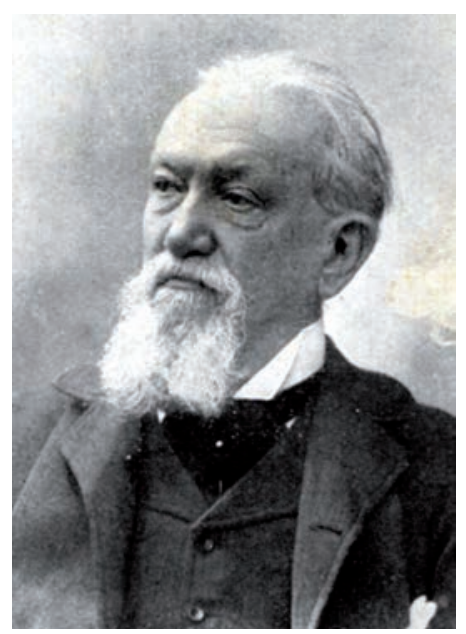

Fig. 14 - Camillo Boito (1836-1914).

Insomma, quando nel dicembre 1863 Brioschi inaugura il Politecnico e l'Accademia scientifico-letteraria, certo dei passi in avanti rispetto alla Legge Casati, nel campo degli studi superiori, sono stati fatti. Al Politecnico non ci sarebbe stata istruzione teologica (sia lode al laicismo dei suoi fondatori), mentre l'assenza di scienze militari e di «un corso di fortificazioni» (Educazione, p. 190) oggi non sono lamentate.

L'ingegneria avrebbe avuto, non una sezione di agronomia, ma per gli ingegneri civili un corso omonimo e un altro di Idraulica fluviale e agricola, nonché un orto botanico, e altre due sezioni meccanica (poi industriale) e normale per i futuri insegnanti di discipline scientifiche. La scuola normale per le materie umanistiche non è oggetto di risarcimento a Pavia ma si istituisce a Milano come Accademia scientifico-letteraria.

La sezione degli architetti civili non ha prevalenti obiettivi del tipo di distinguere (parole di Cattaneo, cit.) «la trabeazione dorica [...] dalla ionica», come grosso modo poteva schematizzarsi dell'Accademia di belle arti, ma fornisce competenze anche tecniche, anche nel settore dell'architettura agraria.

«Il solo ramo di perfezionamento finora istituito è lo studio d'Architettura e Prospettiva per gli Ingegneri-Architetti presso l'Accademia di Belle Arti»(Educazione, p. 117). L'assenza di commenti e di enfasi sembra indicare una scarsa adesione. 
Ancora: se Cattaneo nella proposta di piano di studi per il triennio della Facoltà di Agronomia proponeva corsi di Idraulica agraria, Architettura agraria, Disegno agrario (Educazione, p. 235), al Politecnico l'insegnamento sopra citato di Idraulica fluviale e agricola sarà inizialmente tenuto dallo stesso Brioschi (che già a Pavia aveva insegnato Architettura idraulica, e del quale sono noti interessi e responsabilità anche pubbliche nel settore delle acque). ${ }^{48}$

Il disegno tecnico, creato si può dire - prima alla Società d'Incoraggiamento, poi al Politecnico - da Colombo, non escludeva, tra l'altro, il disegno agrario. Così come Archimede Sacchi, dal 1867 per circa vent'anni, inventa e insegna quel corso di Architettura pratica, che riserva spazio e attenzione (anche nel titolo) all'architettura agraria nel manuale Le abitazioni, alberghi, case operaie, fabbriche rurali, case civili, palazzi e ville (Milano, Hoepli, 1874).

Ma di tutto quanto precede Carlo Cattaneo non sarebbe stato lo stesso soddisfatto, e non solo per la distanza politica rispetto a Brioschi: ma questa è un'altra questione.

48 Cfr. C.A. Fassò, Brioschi e i problemi delle acque, in Francesco Brioschi e il suo tempo (1824-1897), I. Saggi, a cura di C.G. Lacaita e A. Silvestri, Milano, Franco Angeli, 2000. 